\title{
IRE1a deficiency promotes tumor cell death and elF2a degradation through PERK dipendent autophagy
}

\author{
Antonello Storniolo ${ }^{1}$, Vincenzo Alfano ${ }^{2}$, Sabino Carbotta $^{3}$, Elisabetta Ferretti ${ }^{1}$ and Livia Di Renzo ${ }^{1,4}$
}

\begin{abstract}
Sensors of endoplasmic reticulum (ER) stress function in a co-ordinated manner. In the present study we investigated the relationship between IRE1 $a$ and PERK pathways and survival of ER stressed U937 cells and BC3 cells. To this end, we investigated the effects of a subcytotoxic concentration of Tunicamycin in IRE1a-proficient and in IRE1a-deficient cells, by pharmacological inhibition with $4 \mu 8 \mathrm{C}$ or down-regulation by specific siRNA. We show that either type of IRE1 a deficiency affects elF2a expression and causes cell death increase. GSK2606414, a PERK inhibitor, and PERK specific siRNA prevent elF2a down-regulation and restore cell survival. Degradation of this protein is due to autophagy, as it is prevented by bafilomycin and not by proteasome inhibition. Furthermore, activation of the autophagy flux is PERK dependent. Also the Cathepsin B inhibitor CA074 prevents elF2a from degradation and reduces cell death. Altogether, these results show that IRE1a deficiency in ER stressed cells leads to an unexpected decrease of elF2a, an important molecule for protein translation, through PERK dependent autophagy. Thus, IRE1/XBP1 inhibitors may represent a feasible strategy for tumor therapy, while PERK inhibitors may vanish the goal.
\end{abstract}

\section{Introduction}

Most secreted and plasma membrane proteins are folded and matured in the endoplasmic reticulum (ER) lumen. Disturbances in ER calcium homeostasis and protein processing cause the accumulation of misfolded or unfolded proteins in the ER, a cellular condition referred to as "ER stress". Adaptation to ER stress is mediated by the induction of the unfolded protein response (UPR), a regulated and complex signal transduction pathway transmitting information to the cytosol and nucleus to increase protein folding capacity of the $\mathrm{ER}^{1-3}$. The hallmark of the UPR is the upregulation of ER chaperones and folding enzymes, which are required to bind the unfolded proteins and

Correspondence: Livia Di Renzo (livia.direnzo@uniroma1.it)

${ }^{1}$ Department of Experimental Medicine, Sapienza University, 00161 Rome, Italy, Istituto di Ricovero e Cura a Carattere Scientifico Neuromed, Pozzilli, 86077 Isernia, Italy

${ }^{2}$ Department of Molecular Medicine, Sapienza University of Rome, Rome, Italy Full list of author information is available at the end of the article Edited by A Rufini

The original version of this article was revised: following publication of the article, author Elisabetta Ferretti asked for the following institution to be added to her affiliation: Istituto di Ricovero e Cura a Carattere Scientifico Neuromed, Pozzilli, 86077 Isernia, Italy prevent their aggregation ${ }^{4}$. Also a transient attenuation of protein synthesis participates to the UPR by limiting the load of proteins under conditions not well suited to their proper folding, while allowing the transcriptional upregulation of ER chaperones and folding enzymes. ${ }^{5}$. However, cells undergo apoptosis when adaptation mechanisms are unable to alleviate the stress. ${ }^{6,7}$ Thus, the UPR serves to mitigate the stress, or, alternatively, to eliminate stressed cells in order to protect the organism.

Three resident ER transmembrane sensors detect unfolded proteins in the ER to initiate three distinct UPR branches: inositol-requiring protein-1 $\alpha$ (IRE1 $\alpha)$, activating transcription factor-6 (ATF6), and protein kinase RNA (PKR)-like ER kinase (PERK) $)^{3-5,8}$. IRE1 $\alpha$ is an evolutionarily conserved from yeast to human dual enzyme, possessing both a Ser/Thr protein kinase and endoribonuclease activity. Upon BiP/GRP78 (immunoglobulin heavy chain binding protein $/ 78 \mathrm{kDa}$ glucose-regulated protein) dissociation, IRE1 $\alpha$ dimerizes and autophosphorylates, thus, causing a 
conformational change that allosterically activates its endoribonuclease domain. Activated IRE1 $\alpha$, through its RNase domain, excises a $26 \mathrm{bp}$ fragment from the mRNA encoding the transcription factor $\mathrm{X}$-box-binding protein 1 (XBP1) in metazoans, by an unconventional splicing event that leads to generate XBP1s ("s" for spliced), a highly active transcription factor, a key regulator of ER folding capacity, controlling important genes involved in protein quality, ER translocation, glycosylation, and ER/Golgi biogenesis., ${ }^{9,10}$ XBP1 favors cell survival. ${ }^{11}$

PERK phosphorylates the eukaryotic translational initiation factor $2 \alpha$ (eIF2 $\alpha$ ), responsible of reducing protein synthesis and, therefore, the amount of proteins entering the ER. ${ }^{12,13}$ However, despite global translation inhibition, translation of ATF4 (Activating Transcription Factor 4) increases selectively, which upregulates the transcription factor C/EBP-homologous protein $(\mathrm{CHOP})^{14}$. CHOP induction has been linked to apoptosis. ${ }^{15,16}$ It has been also observed that ATF4 and CHOP induce genes involved in autophagy ${ }^{17}$ and the growth arrest and DNA damage-inducible protein GADD34, a protein phosphatase (PP1) targeting protein that directs PP1 to dephosphorylate eIF $2 \alpha^{18,19}$ and, therefore, to allow recovery from protein synthesis shutoff. ${ }^{20}$ It has been reported that PERK $^{-1-}$ cells are hypersensitive to the lethal effects of ER stress. ${ }^{21}$ However, it is also known that

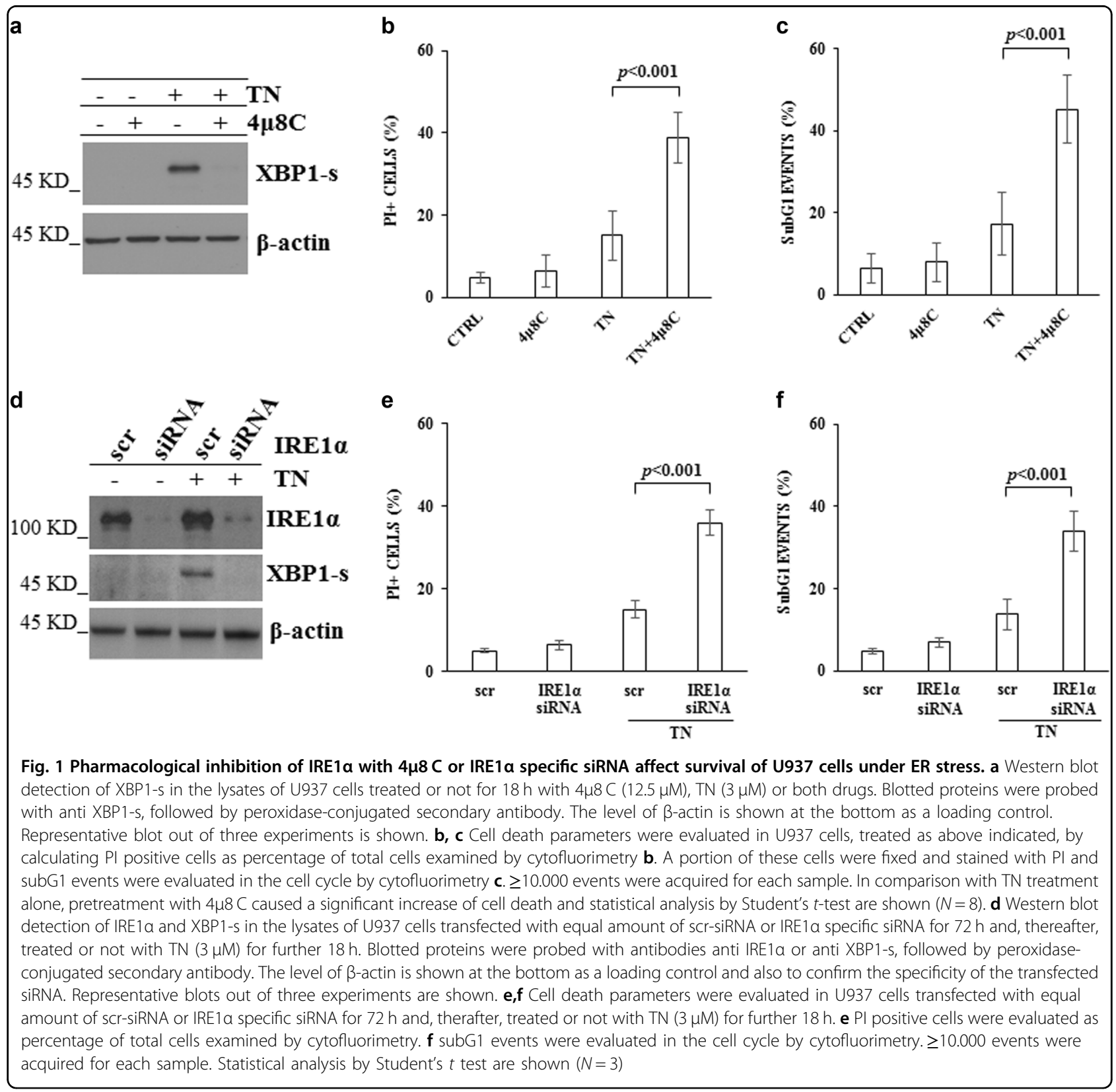


silencing of PERK decreases apoptosis under saturated acid-induced cellular stress. ${ }^{22}$ And also, PERK silencing increases cell viability when ER stress is induced by silver nanoparticles and other data indicate that PERK silencing does not cause more cell death following ER stress. ${ }^{23,24}$ Thus, the role of PERK appears controversial.

Several data have indicated that either IRE1 $\alpha$ or PERKpathway play an important role in controlling autophagyapoptosis crosstalk in ER stressed cells and that both pathways are necessary for the transcriptional upregulation of several autophagy genes. ${ }^{25}$ ER stress sensors function in a co-ordinated manner. IRE1 $\alpha$ and PERK pathways are not independent each other, rather exists a regulatory connection between them. In the present study we set out to investigate the relationship between IRE1 $\alpha$ and PERK pathways and death of ER stressed U937 leukemia cells and BC3 cells, derived from a pleural effusion lymphoma (PEL). To this end, we compared the effects of a subcytotoxic concentration of Tunicamycin (TN), an inhibitor of $N$-linked glycosylation, in IRE1 $\alpha$-proficient and IRE1 $\alpha$-deficient cells, by pharmacological inhibition or specific silencing.

\section{Results}

IRE1 a plays a pro-survival role in cells under ER stress

TN caused death of U937 and BC3 cells in a dosedependent manner (Supplementary Fig. 1a and 1b). ${ }^{26}$ In particular, TN $3 \mu \mathrm{M}$ caused a slight increase of propidium iodide (PI) positive U937 cells $(15 \pm 6 \%)$ in comparison with untreated cultures $(5 \pm 1 \%)$, in addition to the

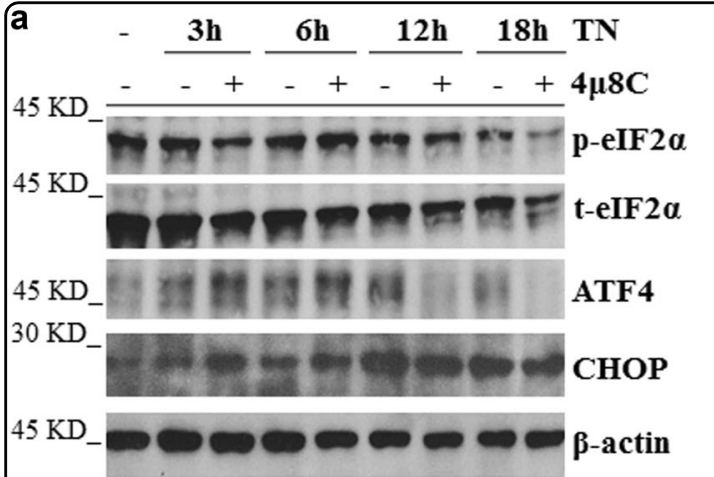

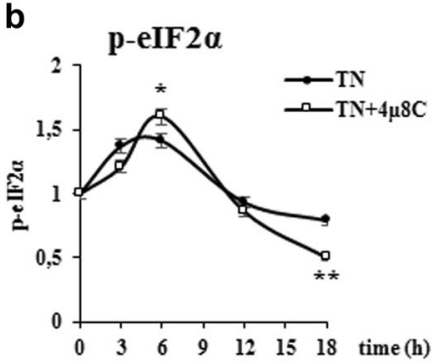
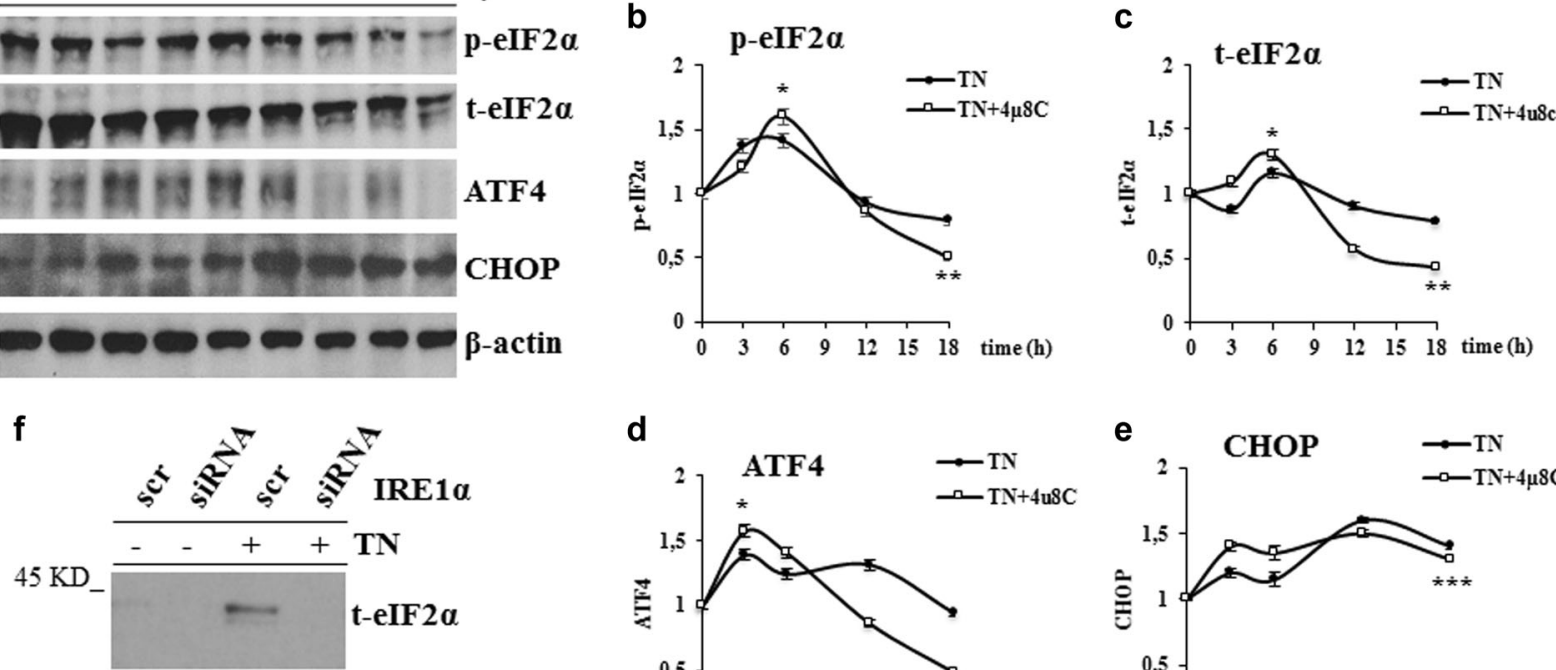

Fold: $1 \quad 0.5 \quad 2.7 \quad 0.5$ $30 \mathrm{KD}$ $=-$ CHOP

Fold: $1 \quad \begin{array}{llll}1 & 0.8 & 2.1 & 1.6\end{array}$

$45 \mathrm{KD}$

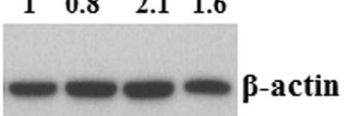

d

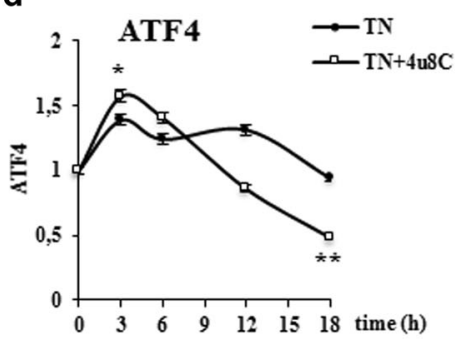

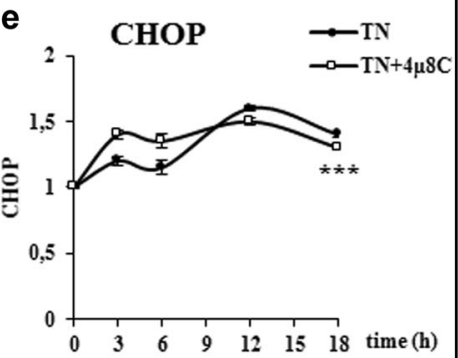

Fig. 2 IRE1a impairment affects elF2a expression. a Representative western blots out of three experiments are shown for p-elF2a, t-elF2a, ATF4 and CHOP detected in the lysates of U937 cells treated for $18 \mathrm{~h}$ with none, TN $(3 \mu \mathrm{M})$ or $4 \mu 8 \mathrm{C}(12.5 \mu \mathrm{M})+\mathrm{TN}(3 \mu \mathrm{M})$ for the indicated hours. Each protein was probed with specific antibody followed by peroxidase-conjugated secondary antibodies. $\beta$-actin is shown as loading control. $\mathbf{b}, \mathbf{c}, \mathbf{d}, \mathbf{e}$ Quantitative analysis of each protein was performed by densitometry of the relative bands in 3 experiments. Values were obtained using the following formula: (densitometry value of the band under examination / densitometry value of the band of the corresponding $\beta$-actin)/(densitometry value of the band under examination in the control / densitometry value of the $\beta$-actin band in the control). Values are means \pm S.D. The statistical differences (Student's $t$-test) between the values obtained in $4 \mu 8 \mathrm{C}+\mathrm{TN}$ cell treatments $\mathrm{v} / \mathrm{s}$ the values obtained in the corresponding TN cell treatments are indicated as: ${ }^{*} P<0.05,{ }^{* *} P<0.01,{ }^{* * *} P>0.05$. f IRE 1 a impairment through specific silencing affects elF2a expression. U937 treated for $72 \mathrm{~h}$ with equal amount of scrambled siRNA or IRE1 a specific siRNA were treated or not with TN $(3 \mu \mathrm{M})$ for $18 \mathrm{~h}$. t-elF2a and CHOP were analyzed by western blot in the cell lysates by specific antibodies followed by peroxidase-conjugated antibodies. $\beta$-actin is shown as loading control and also to confirm the specificity of the transfected siRNA. Values under each band were obtained using the following formula: (densitometry value of the band under examination / densitometry value of the band of the corresponding $\beta$-actin) / (densitometry value of the band of the protein under examination in the lysate of scrambled treated cells / densitometry value of the $\beta$-actin band in the lysate of scrambled treated cells) 
appearance of $17 \pm 8 \%$ subG1 events. Similarly, TN $3 \mu \mathrm{M}$ caused in BC3 cells the appearance of $17 \pm 4 \%$ PI positive cells and $16 \pm 4 \%$ subG1 events, in comparison with $6 \pm$ $1 \%$ PI positive cells and $7 \pm 3 \%$ subG1 events in untreated cultures. SubG1 events were studied by cytofluorimetry of cell cycle phases of cells fixed and then stained with PI. The events preceding the narrow peak of G1 events, and therefore with a hypodiploid content of DNA, are considered to be indicative of apoptotic nuclei. ${ }^{27}$ These results suggest that TN $3 \mu \mathrm{M}$ causes activation of a prosurvival pathway in U937 or BC3 cells. Therefore, this dose was used through the experiments here performed and cell death parameters were investigated after $18 \mathrm{~h}$ treatments.

As TN activates ER stress sensors, we set out to identify IRE1 $\alpha$ role in TN activated survival pathway. Therefore, IRE1 $\alpha$ activation was confirmed by XBP1-s detection in western blotting in U937 cell lysate (Fig. 1a). To be noted, XBP1-s was still present after $18 \mathrm{~h}$ of ER stress, suggesting a cellular need for this protein, whereas IRE1 $\alpha$ pharmacological inhibition with $4 \mu 8 C^{28}$ prevented its expression (Fig. 1a) and caused a significant increase of PI positive cells $(39 \pm 6 \%)$ and subG1 events $(45 \pm 8 \%)$ in TN treated U937 cells (Fig. 1b, c). To further validate the above results, U937 cells were transfected with scr-siRNA or IRE1 $\alpha$-siRNA for $72 \mathrm{~h}$, followed by $18 \mathrm{~h}$ treatment with TN $3 \mu \mathrm{M}$ or none. Knockdown efficiency of IRE1 $\alpha$ was about $85 \%$, still effective after TN cell treatment, and able to prevent XBP1-s expression (Fig. 1d). Furthermore, specific IRE1 $\alpha$ silencing was not cytotoxic per se, whereas it increased cell death after TN treatment (Fig. 1e, f). Similar results were obtained in BC3 cells (Supplementary Fig. 1c and d).

\section{elF2a expression is down-regulated in IRE1 a deficient and ER stressed cells}

The above results suggest that $\mathrm{TN}$ activates IRE1 $\alpha$ playing a pro-survival role and that, upon its impairment, ER stress may be sensed by the other sensors in a prodeath manner. To explore the mechanism causing cell death in IRE1 $\alpha$ deficient cells, we examined in TN and in $4 \mu 8 \mathrm{C}+\mathrm{TN}$ treated U937 cells the expression of proteins of PERK pathway. Thus, p-eIF2 $\alpha$, t-eIF2 $\alpha$, ATF4 and CHOP were analyzed in western blot (Fig. 2a). Densitometric quantification of bands obtained in repeated experiments showed that, in comparison with untreated cells, TN led to a transient increase after $3-6 \mathrm{~h}$ of p-eIF $2 \alpha$ (1.4 fold), after $6 \mathrm{~h}$ of t-eIF2 $\alpha$ ( 1.2 fold), after $3 \mathrm{~h}$ of ATF4 (1.3 fold) and caused after $3-6 \mathrm{~h}$ a slight increase of CHOP, more evident after $18 \mathrm{~h}$, when p-eIF2 $\alpha, \mathrm{t}$-eIF2 $\alpha$ and ATF4 declined to their baseline level (Fig. 2b-e). In comparison with untreated cells, TN together with IRE1 $\alpha$ inhibitor led to a transient larger increase after $6 \mathrm{~h}$ of $\mathrm{p}$ eIF2 $\alpha$ (1.6 fold) and of t-eIF2 $\alpha$ (1.4 fold), and after $3 \mathrm{~h}$ of ATF4 (1.6 fold). This combined treatment led after $3 \mathrm{~h}$ to an increase also of CHOP (1.4 fold). Interestingly, after $12-18 \mathrm{~h}$, the levels of t-eIF2 $\alpha$ and ATF4 became lower (0.5 fold) than in control cells (Fig. 2c, d), while CHOP remained elevated (Fig. 2e). Thus, these results show that either TN or $4 \mu 8 \mathrm{C}+\mathrm{TN}$ treatments cause an initial (within 3-6 h) activation of PERK pathway, followed after $12-18 \mathrm{~h}$ by its decline. However, in comparison with TN treatment, the combined treatment led to an initial significant $(p<0.05)$ increase of t-eIF2 $\alpha$, followed by a significant $(p<0.01)$ decrease, below its constitutive level. ATF4 expression depends on p-eIF2 $\alpha$ and, as expected, paralleled that. Of note, either $\mathrm{TN}$ or the combined treatment with $4 \mu 8 \mathrm{C}+\mathrm{TN}$ caused similar CHOP persistence over time. Similar results were obtained with BC3 cells: in comparison with untreated cells, the combined treatment with $4 \mu 8 \mathrm{C}+\mathrm{TN}$ led after $18 \mathrm{~h}$ to eIF $2 \alpha$ decrease (Supplementary Fig. 2a). In order to further validate these results, t-eIF2 $\alpha$ and CHOP were analyzed by western blot in U937 cells transfected with scr-siRNA or IRE1 $\alpha$-siRNA for $72 \mathrm{~h}$, followed by $18 \mathrm{~h}$ TN treatment or none: in scr-siRNA treated cells t-eIF2 $\alpha$ was well represented and increased 2.1 fold after TN treatment, whereas it appeared already down-regulated in U937 cells transfected with IRE1 $\alpha$-siRNA, even before TN treatment (0.4 and 0.4 fold, respectively). CHOP analysis in the same cell lysates showed that the level of this protein was similarly increased by TN in either scrambled or IRE1 $\alpha$-siRNA treated cells (1.9 and 1.7 fold, respectively) (Fig. 2f).

Thus, IRE1 $\alpha$ impairment in TN stressed cells modifies PERK pathway and, moreover, leads to a decreased expression of eIF2 $\alpha$.

\section{PERK is responsible of elF2 $a$ down-regulation and of the increased death in IRE1a deficient cells, under ER stress}

The above results let us investigate PERK role in the regulation of eIF $2 \alpha$ expression and in the fate of IRE1 $\alpha$ deficient cells, under TN treatment. Thus, U937 cells were pretreated with GSK2606414 (GSK), a PERK inhibitor $^{29,30}$, and thereafter treated or not with TN, $4 \mu 8 \mathrm{C}$, or $4 \mu 8 \mathrm{C}+\mathrm{TN}$. Interestingly, GSK reverted the above results: it blocked t-eIF2 $\alpha$ decrease in IRE1 $\alpha$ deficient U937 cells and it prevented by about 50\% CHOP expression in either IRE1 $\alpha$-deficient or proficient cells (Fig. 3a). Therefore, we examined the role of PERK in deciding the fate of IRE1 $\alpha$-deficient U937 cells, under TN treatment. To this end, cells were pretreated with GSK and thereafter treated with $4 \mu 8 \mathrm{C}$, TN or $4 \mu 8 \mathrm{C}+\mathrm{TN}$. GSK prevented TN and, moreover, $4 \mu 8 \mathrm{C}+\mathrm{TN}$ induced cell death (Fig. 3b, c). Similar results were obtained in BC3 cells pretreated with GSK and thereafter treated with $4 \mu 8$ C, TN or $4 \mu 8 \mathrm{C}+\mathrm{TN}$ (Supplementary Figs. 2A, B and C). Actually, in these cells we observed also an increased expression of $\mathrm{t}$-eIF2 $\alpha$ upon pharmacological inhibition of 


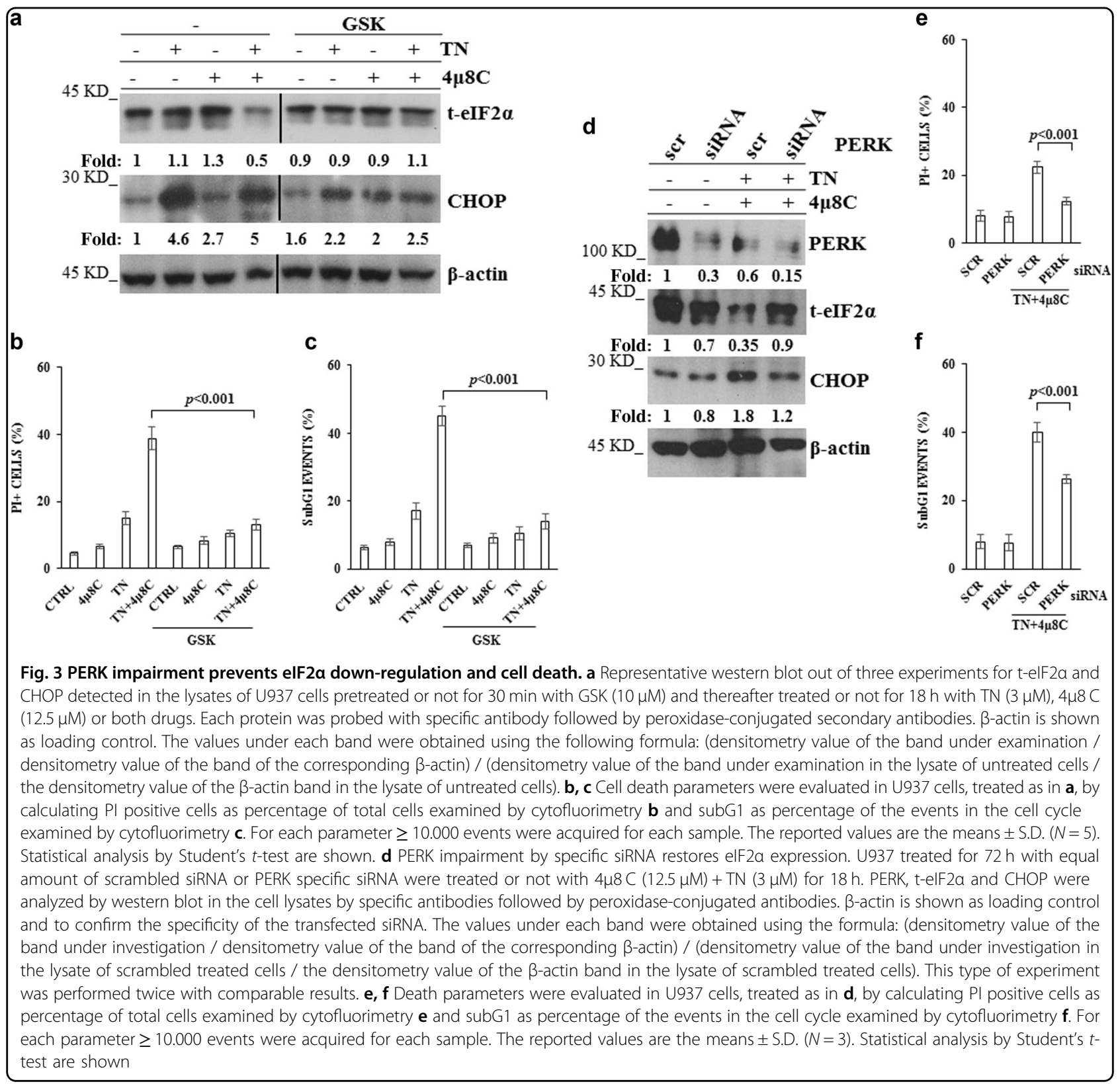

IRE1 $\alpha$ (Supplementary Fig. 2A). To further validate these results PERK expression was silenced in U937 cells by specific siRNA: in comparison with scr-siRNA treated cells, specific silencing caused a decrease of about $70 \%$ of PERK, still evident in $\mathrm{TN}+4 \mu 8 \mathrm{C}$ treated cells (Fig. 3d). Furthermore, PERK-siRNA prevented $4 \mu 8 \mathrm{C}+\mathrm{TN}$ induced t-eIF2 $\alpha$ decrease and CHOP increase (Fig. 3d). Similarly to PERK pharmacological inhibition, also knockdown of this sensor prevented $4 \mu 8 \mathrm{C}+\mathrm{TN}$ induced cell death (Fig. 3e, f). These results confirm a cross-talk between IRE1 $\alpha$ and PERK in ER stressed cells and they show that eIF2 $\alpha$ undergoes an unexpected PERK dependent decrease in IRE1 $\alpha$-deficient cells, that may be relevant for cell survival, being eIF $2 \alpha$ a key molecule at the crossroad of multiple pathways activated in stressed cells, in order to restore cellular homeostasis. Thus, in ER stressed cells, IRE1 $\alpha$ impairment leads to t-eIF $2 \alpha$ decrease and increased cytotoxicity, PERK mediated.

\section{eIF2a is degraded through PERK dependent autophagy}

Quantitative RT-PCR showed that, in comparison with U937 untreated cells, TN induced a 4 fold increase of eIF2 $\alpha$ transcript, that was prevented in a significant manner $(p<0.01)$ by $4 \mu 8 \mathrm{C}$, although the inhibitor per se 


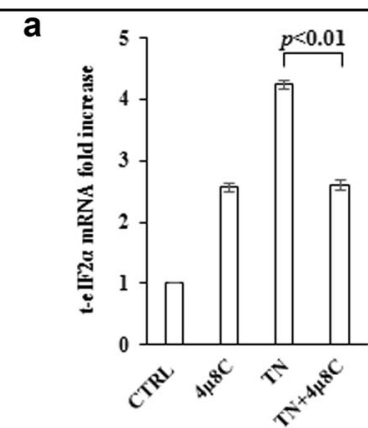

b

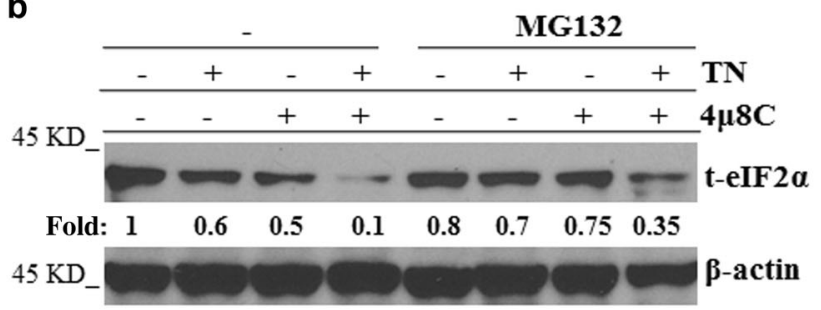

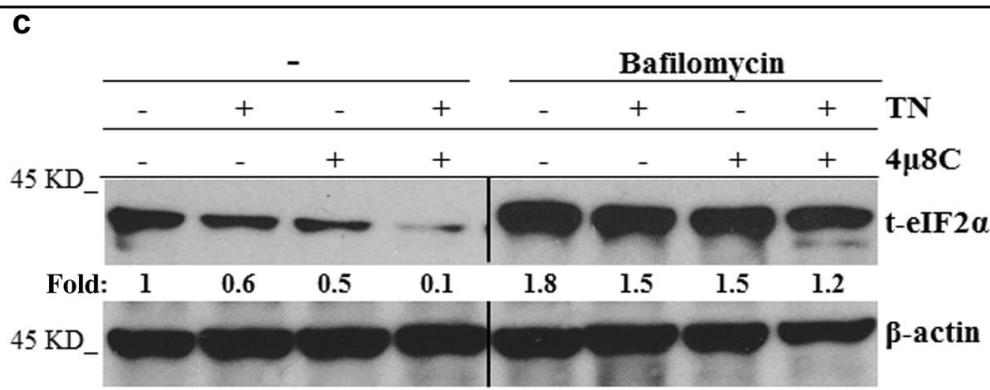

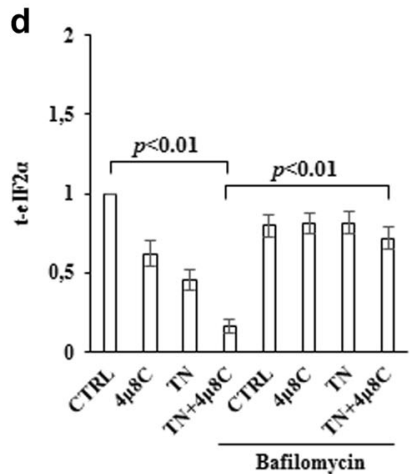

Fig. 4 elF2a expression is restored by bafilomycin and not by proteasome inhibitor. a Quantitative RT-PCR of elF2a mRNA in U937 cells treated or not for $18 \mathrm{~h}$ with $4 \mu 8 \mathrm{C}(12.5 \mu \mathrm{M})$, TN $(3 \mu \mathrm{M})$ or both drugs. The reported values are means \pm S.D. $(N=3)$. Statistical analysis by Student's $t$-test is shown. $\mathbf{b}, \mathbf{c}$ Representative western blots out of three experiments are shown for t-elF2a detected in the lysates of U937 cells treated or not with TN $(3 \mu \mathrm{M}), 4 \mu 8 \mathrm{C}(12.5 \mu \mathrm{M})$ or both drugs for $18 \mathrm{~h}$ and in the last $6 \mathrm{~h}$ treated or not with MG132 (50 $\mu \mathrm{M})$ or with Bafilomycin ( $50 \mathrm{nM})$. $\beta$-actin is shown as loading control. The values under each band were obtained using the formula: (densitometry value of the band under investigation / densitometry value of the band of the corresponding $\beta$-actin) / (densitometry value of the band under investigation in the lysate of untreated cells / the densitometry value of the $\beta$-actin band in the lysate of untreated cells). $\mathbf{d}$ Quantitative analysis of total elF2a detected in the western blots of three independent experiments was performed as above indicated. Results are expressed as mean \pm S.D. and statistical analysis by Student's $t$-test are shown

caused an increase of the transcript (Fig. 4a). Therefore, eIF2 $\alpha$ decrease in $4 \mu 8 \mathrm{C}+\mathrm{TN}$ treated cells depends on transcriptional and post-transcriptional events. Proteasome and autophagy are protein degradative mechanisms. Thus, we inhibited the proteasome with MG132 or autophagy with bafilomycin and analyzed t-eIF2 $\alpha$ by western blot in the lysates of untreated, $4 \mu 8 \mathrm{C}$, TN or $4 \mu 8$ $\mathrm{C}+\mathrm{TN}$ treated cells. MG132 did not restore t-eIF2 $\alpha$ level in $4 \mu 8 \mathrm{C}+\mathrm{TN}$ treated cells, indicating that this protein was not degraded through this route (Fig. 4b). Then, U937 cells were treated or not with $4 \mu 8 \mathrm{C}$, TN or $4 \mu 8 \mathrm{C}+\mathrm{TN}$ for $18 \mathrm{~h}$ and in the last $6 \mathrm{~h}$ were treated or not with bafilomycin to block autophagosomes degradation. ${ }^{31}$ Interestingly, t-eIF2 $\alpha$ levels were restored in bafilomycin treated cells (Fig. 4c, d). These results suggested autophagy involvement in eIF $2 \alpha$ degradation. Thus, we analyzed the autophagy flux and detected that $4 \mu 8 \mathrm{C}$ causes an increase of TN induced flux. In fact, TN caused in $4 \mu 8$ $\mathrm{C}$ treated cells a large increase of LC3-II after block of autophagy degradative steps by bafilomycin (Fig. 5a) and a large decrease of p62 (Fig. 5b, c). U937 cells were also stained with LysoTracker-Red DND99, a fluorescent probe selective for acidic organelles. LysoTracker-Red fluorescence was more pronounced in TN treated cells $(\mathrm{MFI}=13 \pm 2)$ in comparison with untreated or $4 \mu 8 \mathrm{C}$ treated cells $(\mathrm{MFI}=5.5 \pm 0.5$ and $5.6 \pm 0.5$, respectively), while $4 \mu 8 \mathrm{C}+\mathrm{TN}$ caused the largest accumulation of the dye $(M F I=20 \pm 2)$ (Fig. $5 \mathrm{~d}$ ). These results suggest that eIF2 $\alpha$ is actively degraded through the autophagy flux in ER stressed and IRE1 $\alpha$-deficient cells.

To explore PERK role in the autophagy flux causing eIF2 $\alpha$ degradation, western blot analysis of LC3I/II and p62 was performed in the lysates of U937 cells pretreated with GSK and thereafter subjected to ER stress with TN or $4 \mu 8 \mathrm{C}+\mathrm{TN}$. This approach showed that GSK caused LC3-II accumulation and prevented p62 decrease, indicating that PERK was involved in the regulation of the autophagy flux occurring in $\mathrm{TN}$ and in $4 \mu 8 \mathrm{C}+\mathrm{TN}$ treated cells (Fig. 5b, c). Interestingly, GSK pretreatment per se did not modify the fluorescence of untreated cells $(\mathrm{MFI}=5.6 \pm 0.5)$ and of $4 \mu 8 \mathrm{C}$ treated cells $(\mathrm{MFI}=5.6 \pm$ 1 ), while it did not allow the fluorescence development 

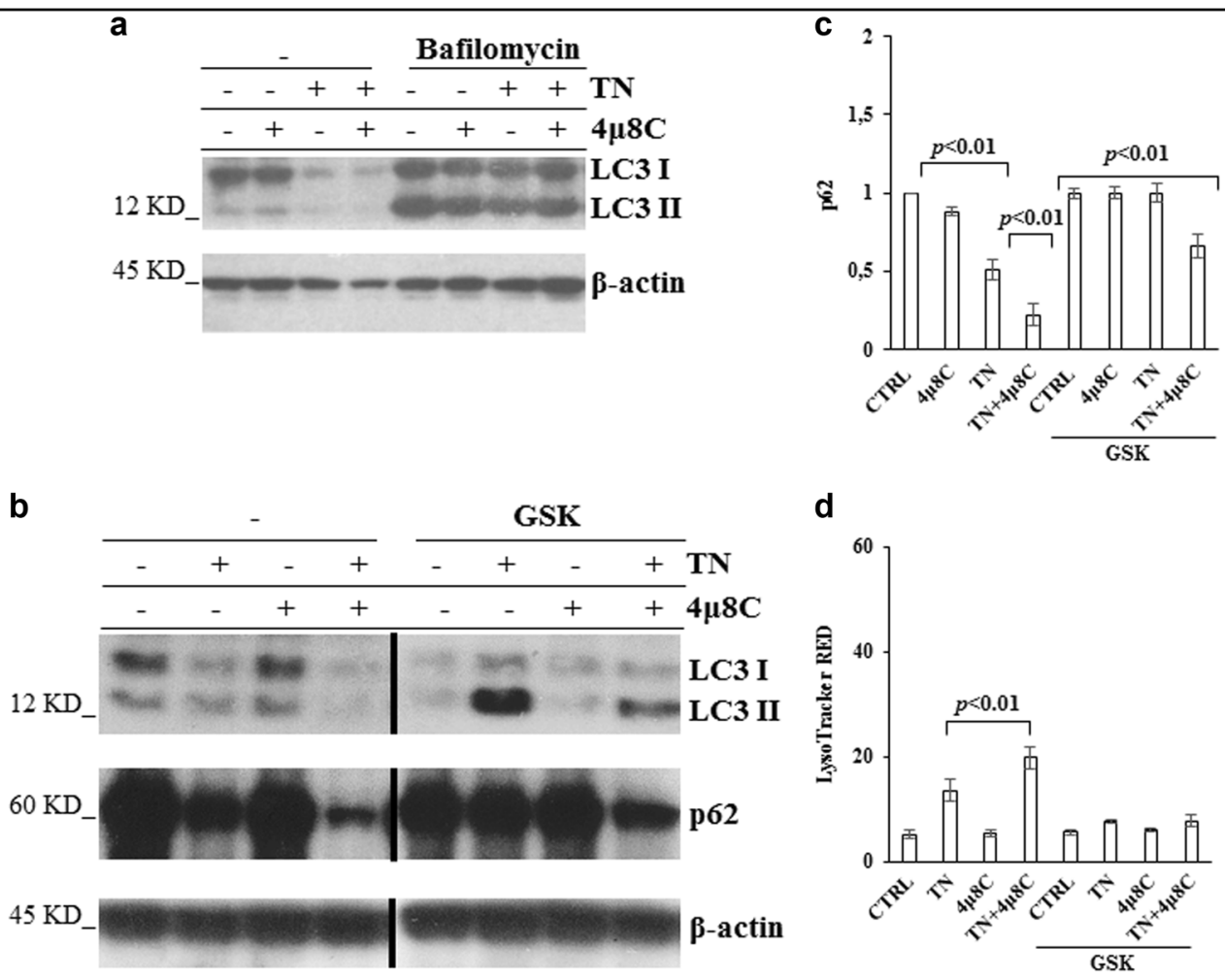

Fig. 5 IRE1 a deficiency causes increased autophagy, PERK dependent. a Representative western blot out of three experiments is shown for LC3I-II detected in the lysates of U937 cells treated or not with $4 \mu 8 \mathrm{C}(12.5 \mu \mathrm{M})$, TN $(3 \mu \mathrm{M})$, or both drugs for $18 \mathrm{~h}$ and in the last $3 \mathrm{~h}$ treated or not with bafilomycin $(50 \mathrm{nM})$. $\beta$-actin is shown as loading control. Specific primary antibodies and peroxidase-conjugated antibodies were used. $\mathbf{b}$ Representative western blots for LC3I-II and p62 detected in the lysates of U937 cells pretreated or not with GSK (10 $\mu \mathrm{M})$ for 30 min and thereafter

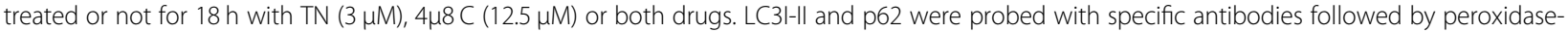
conjugated secondary antibodies. $\beta$-actin is shown as loading control. c Quantitative analysis of p62 was performed by densitometry of the relative bands in the western blots of three experiments. Each value was obtained using the following formula: (densitometry value of the band under examination / densitometry value of the band of the corresponding $\beta$-actin)/(densitometry value of the band under examination in the control / densitometry value of the $\beta$-actin band in the control). The values are means \pm S.D. Statistical differences (Student's $t$-test) are indicated. $\mathbf{d}$ Cells were pretreated or not with GSK $(10 \mu \mathrm{M})$ and then treated or not for $18 \mathrm{~h}$ with TN $(3 \mu \mathrm{M}), 4 \mu 8 \mathrm{C}(12.5 \mu \mathrm{M})$ or both drugs. Thereafter, cells were stained with LysoTracker Red and analyzed by flow cytometry. The Mean Fluorescence Intensities were measured from the histograms representing FL3(log) in $\geq$ 10.000 events. Data are means \pm S.D. of three independent experiments. Statistical analysis by Student's t-test is shown

caused by TN (MFI $=6 \pm 0.5)$ or by $4 \mu 8 \mathrm{C}+\mathrm{TN}(\mathrm{MFI}=$ $6.2 \pm 1$ ) (Fig. 5 d). Similar results were obtained also with BC3 cells, as GSK caused an increased p62 expression (Supplementary Figs. 3A and B) and prevented the LysoTracker-Red fluorescence in $4 \mu 8 \mathrm{C}+\mathrm{TN}$ treated cells (Supplementary Fig. 3C). Thus, IRE1 $\alpha$ impairment causes PERK dependent activation of autophagy leading to eIF2 $\alpha$ degradation.

\section{CA074, a cathepsin B inhibitor, prevents elF2a degradation and cell death}

If eIF2 $\alpha$ degradation was responsible of cell demise following ER stress in IRE1 $\alpha$-deficient cells, inhibition of autophagy should prevent its degradation and, therefore, cell death. However, either 3-MA or bafilomycin or chloroquine addition in the last $6 \mathrm{~h}$ of $18 \mathrm{~h}$ cell treatment with $\mathrm{TN}+4 \mu 8 \mathrm{C}$ did not prevent cell death induced by these drugs (Figs. 6a, b). However, TN-induced ER stress causes disfolded/aggregated proteins and cells need autophagy to degrade them. Therefore, we used lysosome enzymes inhibitors and detected that CA074, a Cathepsin $\mathrm{B}$ inhibitor, added during the last $6 \mathrm{~h}$ of $18 \mathrm{~h}$ treatment with $\mathrm{TN}+4 \mu 8 \mathrm{C}$, prevented cell death (Figs. 6a, b) and, interestingly, prevented eIF $2 \alpha$ decrease in $4 \mu 8 \mathrm{C}+\mathrm{TN}$ treated cells (Fig. 6c).

These results indicate that, in cells under ER stress, IRE1 $\alpha$ deficiency promotes activation of PERK pathway, activating autophagy and eIF2 $\alpha$ degradation through the proteolytic activity of lysosome enzymes and this mechanism is responsible of cell death.

\section{Discussion}

In the present study we show for the first time that eIF2 $\alpha$, an important molecule for protein translation, is 

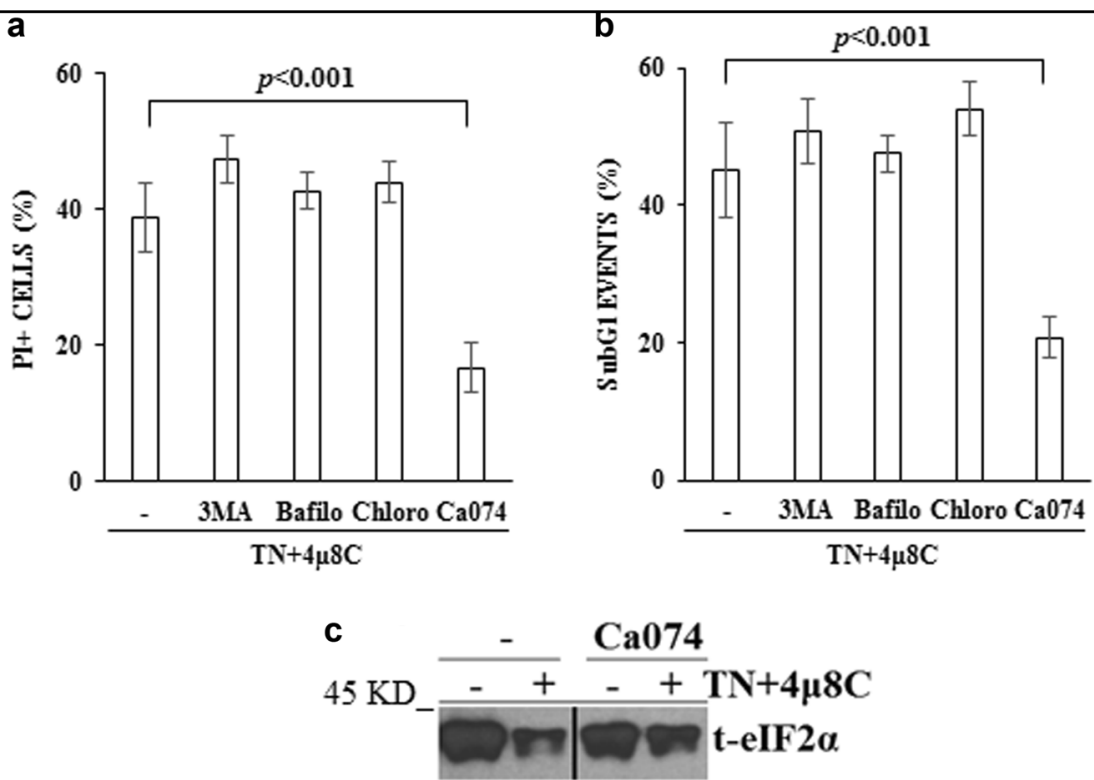

Fold: $1 \quad 0.2 \quad 0.7 \quad 0.8$

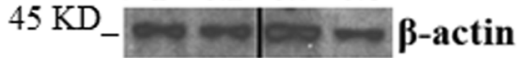

Fig. 6 CA074 prevents elF2a degradation and cell death. a,b U937 cells were treated with $4 \mu 8 \mathrm{C}(12.5 \mu \mathrm{M})+\mathrm{TN}(3 \mu \mathrm{M})$ for $18 \mathrm{~h}$ and in the last $6 \mathrm{~h}$ also with or without 3-MA $(5 \mathrm{mM})$, Bafilomycin $(50 \mathrm{nM})$, Chloroquine $(200 \mu \mathrm{M})$ or CA074 $(10 \mu \mathrm{M})$. Cell death parameters were evaluated by calculating $\mathrm{PI}$ positive cells as percentage of total cells examined by cytofluorimetry $\mathbf{a}$. A portion of these cells were fixed and stained with PI and subG1 events were evaluated in the cell cycle by cytofluorimetry $\mathbf{b}$. For each parameter $\geq 10.000$ events were acquired for each sample. The reported values are means \pm S.D. $(N=3)$. Statistical analysis by Student's $t$-test are shown. c Representative western blot for elF2a detected in the lysates of U937 cells treated or not with $4 \mu 8 \mathrm{C}(12.5 \mu \mathrm{M})+\mathrm{TN}(3 \mu \mathrm{M})$ for $18 \mathrm{~h}$ and with or without CA074 during the last $6 \mathrm{~h}$. $\beta$-actin is shown as loading control. Specific primary antibodies and peroxidase-conjugated antibodies were used. The values under each band were obtained using the following formula: (densitometry value of the band under investigation / densitometry value of the band of the corresponding $\beta$-actin) / (densitometry value of the band under investigation in the lysate of untreated cells / the densitometry value of the $\beta$-actin band in the lysate of untreated cells). This experiment was repeated twice with comparable results

degraded through PERK dependent autophagy in IRE1 $\alpha$ deficient and ER stressed cells.

IRE1 $\alpha$ deficiency was obtained by specific silencing or the pharmacological inhibitor $4 \mu 8 \mathrm{C}$. In both cases, TN promoted an increased cell death and modified the activation of PERK pathway. The investigated proteins belonging to this pathway are eIF2 $\alpha$, ATF4 and CHOP. Unphosphorylated eIF2 $\alpha$ allows protein synthesis, while its phosphorylated form, not allowing protein synthesis, alleviates ER stress. ${ }^{32}$ ATF4 is an important transcription factor, promoting cell survival through activation of genes involved in aminoacid metabolism, redox reactions and protein secretion. ${ }^{33}$ CHOP is a transcription factor, playing a pro-apoptosis role. ${ }^{15,16}$

The activation of PERK pathway here observed can be examined at early and late times, i.e. at $3-6 \mathrm{~h}$ and at $18 \mathrm{~h}$ of cell treatment with TN with or without the pharmacological inhibitor $4 \mu 8 \mathrm{C}$. Indeed, in comparison with IRE1 $\alpha$-proficient cells, eIF2 $\alpha$ and ATF4 appeared increased in the first 3-6 h of IRE1 $\alpha$ inhibition, while they decreased thereafter, below their respective constitutive levels, whereas CHOP expression was only slightly affected in IRE1 $\alpha$-deficient cells. The late results were obtained also after IRE1 $\alpha$ silencing, thus, rouling out possible off-target effects of the inhibitor.

ATF4 down-regulation in IRE1 $\alpha$-deficient cells paralleled that of t-eIF $2 \alpha$, thus, supporting that its expression during ER stress is linked to eIF2 $\alpha$. Also CHOP could be expected to be strongly down-regulated, being its translation under ATF4 control. However, this was not the case and could depend on the half-life of this protein, but also on the fact that CHOP may be transcribed under the activity of ATF6, another sensor of ER stress. ${ }^{34}$ However, the similar expression of CHOP in IRE1 $\alpha$-proficient and -deficient cells suggests that it is not responsible of the increased cell death observed in IRE1 $\alpha$ deficient cells.

These results indicate the existence of a molecular cross-talk between ER stress sensors, where IRE1 $\alpha$ controls PERK pathway. Future studies will explain how IRE1 $\alpha$ controls PERK and its pathway.

In this study we investigated the molecular terms leading to eIF2 $\alpha$ down-regulation and found that it 
depended on PERK, as inhibition or specific siRNA of this sensor prevented eIF $2 \alpha$ decline in IRE1 $1 \alpha$-deficient cells. In the same cells CHOP was only in part increased, and cell death was prevented. These results confirm the link between PERK and CHOP expression and suggest a new mechanism linking PERK and eIF2 $\alpha$, as this protein, in IRE1 $\alpha$ absence, would be down-regulated under PERK control.

Protein decrease can be due to decreased transcription and/or increased degradation. For what concerns eIF2 $\alpha$, we observed a partial decrease of its mRNA in cells treated with $4 \mu 8 \mathrm{C}+\mathrm{TN}$, in comparison with cells treated only with TN. This finding suggests that IRE1 $\alpha$ contributes to the regulation of the transcription of eIF $2 \alpha$, without excluding not transcriptional mechanisms. Thus, we examined cell machineries degrading proteins. The proteasome was inhibited with MG132, while the autophagy flux was blocked with bafilomycin: eIF $2 \alpha$ expression was restored to normal levels only upon autophagy inhibition. Of interest, in comparison with TN treatment, $4 \mu 8 \mathrm{C}+\mathrm{TN}$ caused a more conspicous increase of the acidic compartment, as shown by the LysoTracker Red fluorescence, a larger decrease of p62, known to be degraded in the final steps of autophagy, and an increased degradation of LC3-II, as indicated by bafilomycin experiments. Thus, all together, IRE1 $\alpha$ deficiency leads ER stressed cells to eIF2 $\alpha$ degradation through a more sustained autophagy flux.

It is known that IRE1 $\alpha / \mathrm{XBP} 1$ deficiency leads to augmented autophagy, enhancing the clearance of the mutant superoxide dismutase-1 (SOD1) protein. ${ }^{35}$ However, in those studies the relationship between IRE1 $\alpha$ and PERK was not investigated, while our results indicate a link between IRE1 $\alpha$ deficiency and PERK dependent activation of autophagy.

It is known that eIF2 $\alpha$ and ATF4, under PERK activation, are responsible of $L C 3$ transcription and autophagy activation. ${ }^{36}$ And, indeed, we observed that either TN or $\mathrm{TN}+4 \mu 8 \mathrm{C}$ activate autophagy through PERK involvement. In fact, GSK prevented the decrease of LC3-II and of p62 following TN $+4 \mu 8 \mathrm{C}$ cell treatment. These findings show PERK involvement in autophagy regulation and, therefore, in eIF2 $\alpha$ degradation and they suggest a relationship between intensity of PERK-pathway activation and intensity of autophagy activation, even if the increase of eIF2 $\alpha$ and ATF4 occurring in the initial $6 \mathrm{~h}$ of ER stress should be responsible of the increased autophagy flux still occurring after $12-18 \mathrm{~h}$. However, it would be also possible that PERK enters into autophagy activation through different and, eventually, selective mechanisms, without the involvement of eIF2 $\alpha /$ ATF 4 pathway.

Autophagy is responsible of degradation of proteins and organelles by lysosome enzymes in order to maintain cellular omeostatic conditions. In this way, cells under ER stress degrade aggregated proteins and survive. However, conditions causing too long or excessive autophagy may lead to cell death. ${ }^{37,38}$ Here we observed that in ER stressed and IRE1 $\alpha$-deficient cells inhibition of autophagy by 3-MA or chloroquine or bafilomycin was slightly more cytotoxic. Interestingly, CA074 did not allow eIF $2 \alpha$ degradation and increased cell survival. This inhibitor blocks a portion of lysosome enzymes, like Cathepsin B, leaving other enzymes still efficient in degrading the autophagosome cargo. Thus, the cells would survive, as they benefit of the ongoing autophagy flux and the presence of eIF $2 \alpha$.

In aminoacid starvation, proteins involved in translation, such as ribosomal proteins and translation initiation factors, undergo a pronounced degradation..$^{39}$ Ribosomes appear to be stable within $5 \mathrm{~h}$ of starvation and to be degraded specifically by autophagy in long term starvation. Thus, it would be possible that also eIF $2 \alpha$ undergoes degradation through autophagy at a late time, i.e. around 12-18 h, of ER stress in IRE1 $\alpha$ deficient cells. Whether this condition would resemble amino-acid starvation or represents a new condition of autophagy activation remains to be investigated.

Altogether, the results here reported reinforce the possibility that IRE1 $\alpha / \mathrm{XBP} 1$ inhibitors, by causing eIF2 $\alpha$ degradation, may represent a strategy for tumor treatment and, moreover, these results indicate that the increased autophagy is PERK dependent and, therefore, that conditions affecting this sensor may vanish the proposed goal.

\section{Materials and methods \\ Materials}

Antibody anti-SQSTM1/p62 was from BD Transduction Laboratories (San Jose, CA, USA). Antibodies anti-BiP, -CHOP, phoshorylated-eIF2 $\alpha$ (p-eIF2 $\alpha$ ), total-eIF2 $\alpha$ (t-eIF2 $\alpha),-$ IRE1 $\alpha,-$ LC3I/II and horseradish peroxidase (HRP)-conjugated anti-rabbit- and anti-mouseimmunoglobulin antibodies were from Cell Signaling Technology (Danvers, MA, USA). Antibody anti- $\beta$ actin, bafilomycin, bovine serum albumin (BSA), chloroquine, HBSS, l-glutamine, 3-methyladenine, penicillin-streptomycin, phosphate buffered saline (PBS), PI, RNAse, and RPMI-1640 were from Sigma-Aldrich (St. Louis, MO, USA). Fetal calf serum (FCS) (GIBCO), LysoTracker-Red, Lipofectamine RNAiMAX and OPTI-MEM medium (GIBCO), siRNA-IRE1 (Ambion), siRNA-PERK (Ambion) and scrambled siRNA (Ambion) were from Life Technologies (Invitrogen, San Giuliano Milanese, Italy). RC-DC protein assay, SDS-sample buffer, protein standard, SDSPAGE reagents and polyvinylidene difluoride membranes were from Bio-Rad Laboratories (Segrate, Italy). ECL fast femto was from Società Italiana Chimici (Rome, Italy). TN, $4 \mu 8 \mathrm{c}$ and GSK2606414 were from Calbiochem (San Diego, CA, USA). 


\section{Cells and cell viability}

U937 cells, a human monoblastic leukemia cell line, and BC3 cells, a human B-cell line derived from a PEL carrying latent Kaposi's Sarcoma-associated Herpes Virus (KSHV), were grown in RPMI-1640 medium supplemented with 5\% heat-inactivated FCS, $2 \mathrm{mM}$ glutamine, 100 units $/ \mathrm{ml}$ penicillin and $100 \mu \mathrm{g} / \mathrm{ml}$ streptomycin, at $37^{\circ} \mathrm{C}$, in fully humidified $95 \%$ room air $/ 5 \% \mathrm{CO}_{2}$. Cells were resuspended three times a week in fresh complete medium to $3 \times 10^{5}$ / ml.Cells in every experiment were $\geq 94 \%$ viable, as assessed by calculating alive trypan blue-excluding cells as percentage of all cells counted. They were washed, resuspended in complete medium, $1 \times 10^{6} / \mathrm{ml}$, transferred to multi-well plates and then treated with inhibitors or vehicles, incubated for $30 \mathrm{~min}$, and subsequently exposed to test agents or, again, to vehicles. At the end of each experiment, cells were gently mixed and aliquots taken for PI staining and cell cycle analysis. Vehicles, even when used in combination, were $\leq 0.3 \%(\mathrm{v} / \mathrm{v})$ and did not modify any investigated parameter in comparison with control cultures.

\section{Flow cytometry analysis of cell death}

Nuclear DNA fragmentation was quantified by flow cytometry evaluation of hypodiploic (subG1) DNA events after cell fixation and PI staining. ${ }^{27,40}$ Briefly, cells were washed with PBS, pelletted and fixed in ice cold ethanol/ water $(70 / 30, \mathrm{v} / \mathrm{v})$ for $1 \mathrm{~h}$, pelletted again, washed twice with PBS, and finally resuspended in PBS containing RNAse $(20 \mu \mathrm{g} / \mathrm{ml})$ and PI $(100 \mu \mathrm{g} / \mathrm{ml})$. Events in the different cell cycle phases were gated manually using an EPICS XL cytofluorimeter (Beckman Coulter, Hialeah, Fl, USA). At least 10.000 events/sample were acquired. Collected data were analyzed using the Multicycle software for DNA content and cell cycle analysis (Phoenix Flow System, San Diego, CA, USA). SubG1 events, representative of apoptotic cells, are given as percentage of the total cell population.

Membrane permeability, indicative of cell death, was investigated by resuspending the cells in HBSS containing PI $(20 \mu \mathrm{g} / \mathrm{ml})$ at room temperature and analyzed by flow cytometry (EPICS-XL), measuring the fluorescence emission at $>575 \mathrm{~nm}$ (FL3log).

\section{Western blot analysis}

Whole cell lysates were prepared as previously described. ${ }^{26}$ Briefly, the cells were kept for $30 \mathrm{~min}$ on ice in lysis buffer $(\mathrm{NaCl} 150 \mathrm{mM}, \mathrm{CaCl} 21 \mathrm{mM}, \mathrm{MgCl} 21 \mathrm{mM}, \mathrm{NaN} 3$ $0.1 \%, \mathrm{NaF} 10 \mathrm{mM}$, Triton X-100 1\% (v/v), orthovanadate 1 $\mathrm{mM}$, aprotinin $2 \mu \mathrm{g} / \mathrm{ml}$, leupeptin $2 \mu \mathrm{g} / \mathrm{ml}$, iodoacetamide $10 \mathrm{mM}$, PMSF $2 \mathrm{mM}$, and pepstatin $20 \mu \mathrm{M}$ ). Appropriate volumes of $4 \mathrm{x}$ SDS-sample buffer $(\mathrm{v} / \mathrm{v})$ were then added. Cell lysates were briefly sonicated, warmed at $95^{\circ} \mathrm{C}$ for 5 min, and cleared by $14.000 \mathrm{x}$ g centrifugation in a microfuge for $15 \mathrm{~min}$ at $4^{\circ} \mathrm{C}$. Supernatants were collected and proteins were quantified by RC-DC protein assay. Equal protein amounts were separated from the different samples by SDS-PAGE and blotted onto PVDF membranes. Transfer efficiency was checked with Ponceau staining. Blots, blocked in tris-buffered saline containing BSA 5\% $(\mathrm{w} / \mathrm{v})$, were probed with specific primary antibodies, washed with PBS-Tween-20, and then incubated with peroxidase-conjugated secondary antibody. Finally, each membrane was probed to detect $\beta$-actin. The final dilutions and incubation times suggested by the manufacturer were used for each antibody. Immunodetection was performed using the ECL reagents. Densitometry quantitation of the bands was performed using ImageJ software (National Institutes of Health, Bethesda, MD, USA) on a Mac OS X (Apple Computer International, Cupertino, CA, USA).

\section{SiRNA}

RNA knockdown was performed with pools of siRNA duplexes. Briefly, cells were washed and resuspended in OPTI-MEM medium, transfected with siRNA specific for human IRE1 $\alpha$ or for human PERK and relative scrambled siRNA (Ambion), using lipofectamine RNAiMAX according to the manufacturer's guidelines and as we previously described. ${ }^{41}$ After $12 \mathrm{~h}$ of incubation, RPMI1640 containing $20 \%$ fetal calf serum was added without removing the transfection medium. The cells were cultured for further $60 \mathrm{~h}$. After centrifugation, the medium was replaced with fresh RPMI-1640, containing 10\% fetal calf serum, and the cells cultured again in the presence or not of $\mathrm{TN}$ or $4 \mu 8 \mathrm{C}+\mathrm{TN}$.

\section{RNA isolation and qRT-PCR}

Unless otherwise indicated, reagents and equipment were purchased from Thermo Fisher Scientific. Total RNA was purified using Trizol. One $\mu \mathrm{g}$ was reverse transcribed using random primers and SuperScript II. Quantitative RT-PCR (qRT-PCR) analysis was performed using the ViiA 7 Real-Time PCR System, using the "best coverage" TaqMan gene expression assays, specific for each analyzed mRNA, according to manufacturer's protocol. Each amplification reaction was performed in triplicate, and the average of the three threshold cycles was used to calculate the amount of transcripts in the sample (SDS 2.3 software). mRNA quantification was expressed, in arbitrary units, as the ratio of the sample quantity to the calibrator or to the mean values of control samples. All data are normalized to the mean value of three endogenous controls: GusB, $\beta 2$-microglobulin and HPRT.

\section{Acidic compartment evaluation by LysoTracker Red}

Cells were incubated for $15 \mathrm{~min}$ in HBSS containing LysoTracker-Red DND-99 (100 nM), a fluorescent probe with high selectivity for acidic organelles. HBSS washed 
cells were analyzed in a cytofluorimeter EPICS XL. Under cytofluorimetry, control and treated cells showed similar side- and forward-scatter characteristics and the gate used for each sample was the same as in control cells. The red fluorescence intensity of the gated cells was analyzed on a $\log$ scale (FL3log) and recorded as Mean Fluorescence Intensity (MFI). A minimum of 10.000 events were examined for each sample. The results are expressed according to the formula (MFI in treated cells)/(MFI in control cells)x 100 or (MFI in siRNA cells)/(MFI in scrRNA cells) $\mathrm{x} 100$.

\section{Statistical analysis}

Results are expressed as mean \pm S.D. of repeated experiment, as indicated in the figure legends. Statistical differences between the data sets were evaluated using unpaired, two-tailed Student's $t$-test. Values of $p<0.05$ were considered statistically significant.

\section{Acknowledgements}

This work was supported by Ateneo grants (2014 and 2016) from University La Sapienza of Rome to LDR.

\section{Author details}

${ }^{1}$ Department of Experimental Medicine, Sapienza University, 00161 Rome, Italy, Istituto di Ricovero e Cura a Carattere Scientifico Neuromed, Pozzilli, 86077 Isernia, Italy. ${ }^{2}$ Department of Molecular Medicine, Sapienza University of Rome, Rome, Italy. ${ }^{3}$ Department of Surgical Sciences, Sapienza University of Rome, Rome, Italy. ${ }^{4}$ Azienda Ospedaliera Universitaria Policlinico Umberto I, Rome, Italy

\section{Author contributions}

AS performed most of the experiments and analyzed the results. VA performed the rtPCR. EF and SC participated in the analysis of the results. LDR designed the overall study and supervised the work. All authors discussed the results and made substantial contributions to the manuscript.

\section{Competing interests}

The authors declare that they have no competing interests.

Publisher's note: Springer Nature remains neutral with regard to jurisdictional claims in published maps and institutional affiliations.

\section{Supplementary information}

The online version of this article (https://doi.org/10.1038/s41420-017-0002-9) contains supplementary material.

Received: 14 September 2017 Accepted: 29 September 2017 Published online: 29 January 2018

\section{References}

1. Kaufman, R. J. Stress signaling from the lumen of the endoplasmic reticulum: coordination of gene transcriptional and translational controls. Genes Dev. 13, 1211-1233 (1999).

2. Harding, H. P., Calfon, M., Urano, F., Novoa, I. \& Ron, D. Transcriptional and translational control in the mammalian unfolded protein response. Annu. Rev. Cell. Dev. Biol. 18, 575-599 (2002).

3. Ron, D. \& Walter, P. Signal integration in the endoplasmic reticulum unfolded protein response. Nat. Rev. Mol. Cell. Biol. 8, 519-529 (2007).

4. Harding, H. P. et al. Translational regulation in the cellular response to biosynthetic load on the endoplasmic reticulum. Cold Spring Harb. Symp. Quant. Biol. 66, 499-508 (2001).
5. Schröder, M. \& Kaufman, R. J. The mammalian unfolded protein response. Annu. Rev. Biochem. 74, 739-789 (2005).

6. Szegezdi, E., Logue, S. E., Gorman, A. M. \& Samali, A. A. Mediators of endoplasmic reticulum stress-induced apoptosis. EMBO. Rep. 7, 880-885 (2006).

7. Xu, C., Bailly-Maitre, B. \& Reed, J. C. Endoplasmic reticulum stress: cell life and death decisions. J. Clin. Invest. 115, 2656-2664 (2002).

8. Urano, F., Bertolotti, A. \& Ron, D. IRE1 and efferent signaling from the endoplasmic reticulum. J. Cell. Sci. 113, 3697-3702 (2000).

9. Lee, K. et al. IRE1-mediated unconventional mRNA splicing and S2P-mediated ATF6 cleavage merge to regulate XBP1 in signaling the unfolded protein response. Genes Dev. 16, 452-466 (2002).

10. Yoshida, H., Matsui, T., Yamamoto, A., Okada, T. \& Mori, K. XBP1 mRNA is induced by ATF6 and spliced by IRE1 in response to ER stress to produce a highly active transcription factor. Cell 107, 881-891 (2001).

11. Lin, J. $H$. et al. IRE1 signaling affects cell fate during the unfolded protein response. Science 318, 944-949 (2007).

12. Shi, $Y$. et al. Identification and characterization of pancreatic eukaryotic initiation factor 2 alpha-subunit kinase, PEK, involved in translational control. Mol. Cell. Biol. 18, 7499-7509 (1998)

13. Harding, H. P., Zhang, Y. \& Ron, D. Protein translation and folding are coupled by an endoplasmic-reticulum-resident kinase. Nature. 397, 271-274 (1999).

14. Lu, P. D., Harding, H. P. \& Ron, D. Translation reinitiation at alternative open reading frames regulates gene expression in an integrated stress response. J. Cell. Biol. 167, 27-33 (2004).

15. Wang, X. Z. et al. Signals from the stressed endoplasmic reticulum induce C/ EBP-homologous protein (CHOP/GADD153). Mol. Cell. Biol. 16, 4273-4280 (1996).

16. Marciniak, S. J. et al. CHOP induces death by promoting protein synthesis and oxidation in the stressed endoplasmic reticulum. Genes Dev. 18, 3066-3077 (2004).

17. B'chir, W. et al. The elF2/ATF4 pathway is essential for stress-induced autophagy gene expression. Nucleic Acid Res 41, 7683-7699 (2013).

18. Brush, M. H., Weiser, D. C. \& Shenolikar, S. Growth arrest and DNA damageinducible protein GADD34 targets protein phosphatase 1 a to the endoplasmic reticulum and promotes dephosphorylation of the a subunit of eukaryotic translation initiation factor 2. Mol. Cell. Biol. 23 , 1292-1303 (2003)

19. Connor, J. H., Weiser, D. C., Li, S., Hallenbeck, J. M. \& Shenolikar, S. Growth arrest and DNA damage-inducible protein GADD34 assembles a novel signaling complex containing protein phosphatase 1 and inhibitor 1. Mol. Cell. Biol. 21, 6841-6850 (2001).

20. Kojima, E. et al. The function of GADD34 is a recovery from a shutoff of protein synthesis induced by ER stress: elucidation by GADD34-deficient mice. FASEB. J. 17, 1573-1575 (2003).

21. Harding, H. P., Zhang, Y., Bertolotti, A., Zeng, H. \& Ron, D. PERK is essential for translational regulation and cell survival during the unfolded protein response. Mol. Cell. 5, 897-904 (2000).

22. Cao, J. et al. Saturated fatty acid induction of endoplasmic reticulum stress and apoptosis in human liver cells via the PERK/ATF4/CHOP signaling pathway. Mol. Cell. Biochem. 364, 115-129 (2012).

23. Zhang, R. et al. Endoplasmic reticulum stress signaling is involved in silve nanoparticles-induced apoptosis. Int. J. Biochem. Cell. Biol. 44, 224-232 (2012).

24. Walter, F., Schmid, J., Düssmann, H., Concannon, C. G. \& Prehn, J. H. Imaging of single cell responses to ER stress indicates that the relative dynamics of IRE1/ XBP1 and PERKVATF4 signalling rather than a switch between signalling branches determine cell survival. Cell. Death. Differ. 22, 1502-1516 (2015).

25. Deegan, S. et al. A close connection between the PERK and IRE arms of the UPR and the transcriptional regulation of autophagy. Biochem. Biophys. Res. Commun. 456, 305-311 (2015).

26. Raciti, M., Lotti, L. V., Valia, S., Pulcinelli, F. M. \& Di Renzo, L. JNK2 is activated during ER stress and promotes cell suival. Cell Death Dis 3, e429 (2012).

27. Nicoletti, I., Migliorati, G., Pagliacci, M. C., Grignani, F. \& Riccardi, C. A rapid and simple method for measuring thymocyte apoptosis by propidium iodide staining and flow cytometry. J. Immunol. Methods. 139, 271-279 (1991).

28. Cross, B. C. et al. The molecular basis for selective inhibition of unconventional mRNA splicing by an IRE1-binding small molecule. Proc Natl Acad Sci USA 109, E869-E878 (2012).

29. Axten, J. M. et al. Discovery of 7-methyl-5-(1-\{3-(trifluoromethyl)phenyl]acetyl\}2,3-dihydro-1H-indol-5-yl)-7H-pyrrolo[2,3-d]pyrimidin-4-amine (GSK2606414), a potent and selective first-in-class inhibitor of protein kinase $R$ (PKR)-like endoplasmic reticulum kinase (PERK). J. Med. Chem. 55, 7193-7207 (2012). 
30. Atkins, $C$. et al. Characterization of a novel PERK kinase inhibitor with antitumor and antiangiogenetic activity. Cancer. Res. 73, 1993-2002 (2013).

31. Klionsky, D. J. et al. Guidelines for the use and interpretation of assays for monitoring autophagy (2nd edition). Autophagy. 12, 1-222 (2016).

32. Wek, R. C. \& Anthony, T. G. Extending beta cell survival by UPRegulating ATF4 translation. Cell. Metab. 4, 333-334 (2006).

33. Harding, H. P. et al. An integrated stress response regulates aminoacid metabolism and resistance to oxidative stress. Mol. Cell. 11, 619-633 (2003).

34. Hirsch, I., Weiwad, M., Prell, E. \& Ferrari, D. M. ERp29 deficiency affects sensitivity to apoptosis via imapirment of the ATF6-CHOP pathway of stress response. Apoptosis. 19, 801-815 (2014).

35. Hetz, C. et al. XBP-1 deficiency in the nervous system protects against amyotrophic lateral sclerosis by increasing autophagy. Genes Dev. 23 2294-2306 (2009).

36. Rzymski, T. et al. Regulation of autophagy by ATF4 in response to severe hypoxia. Oncogene. 29, 4424-4435 (2010).
37. Moretti, I., Cha, Y. I., Niermann, K. J. \& Lu, B. Switch between apoptosis and autophagy: radiation-induced endoplasmic reticulum stress? Cell Cycle $\mathbf{6}$ 793-798 (2007).

38. Kröemer, G., Mariño, G. \& Levine, B. Autophagy and the integrated stress response. Mol. Cell. 40, 280-293 (2010).

39. Gretzmeier, C. et al. Degradation of protein translation machinery by amino acid starvation-induced macroautophagy. Autophagy. 13, 1064-1075 (2017).

40. Matusali, G., Arena, G., De Leo, A., Di Renzo, L. \& Mattia, E. Inhibition of p38 MAP kinase pathway induces apoptosis and prevents Epstein Barr virus reactivation in Raji cells exposed to lytic cycle inducing compounds. Mol. Cancer. 8, 18 (2009).

41. Storniolo A., Raciti M., Cucina A., Bizzarri M. \& Di Renzo L. Quercetin affects Hsp70/IRE1a mediated protection from death induced by endoplasmic reticulum stress. Oxid. Med. Cell. Longev. 2015: 645157 1-11 (2015). 